\title{
Where do the immune checkpoint inhibitors stand in the management of triple negative breast cancer?
}

\author{
Elie El Rassy*,1, Fadi El Karak ${ }^{1}$ \& Marwan Ghosn ${ }^{1}$ \\ ${ }^{1}$ Department of Medical Oncology, Hotel Dieu de France University Hospital, Faculty of Medicine, Saint Joseph University, Beirut, \\ Lebanon \\ * Author for correspondence: elie.rassy@hotmail.com
}

' $\mathrm{ICl}$-based combinations with chemotherapy, novel targeted agents and other immunotherapies would reduce the activity of T-regulatory cells, enhance cross-penetration of tumor antigens and increase PD-L1 expression and infiltration of $\mathrm{CD}^{+}$T cells."

First draft submitted: 20 November 2017; Accepted for publication: 14 December 2017; Published online: 9 February 2018

Keywords: early stage $\bullet$ immune checkpoint inhibitors $\bullet$ locally advanced $\bullet$ metastatic $\bullet$ triple negative breast cancer

Breast cancer is a heterogeneous disease defined by the expression of the hormone receptors and the HER2. The subtype triple negative breast cancer (TNBC) constitutes $15-20 \%$ of breast cancer and is defined by the lack the expression of any of these biomarkers [1]. It is associated with a younger age and advanced stage at diagnosis, increased risk of visceral metastasis and poorer outcome [2]. As such, it remains a therapeutic challenge in medical practice. Unlike other molecular subtypes that present targetable drivers, TNBC does not benefit from any targeted drug. The current standard-of-care treatment of TNBC is chemotherapy in the adjuvant, neoadjuvant and metastatic settings. TNBC is highly sensitive to chemotherapy in comparison to luminal breast cancers with pathologic complete response (pCR) rating 30-40\% versus $10-25 \%$, respectively after neoadjuvant chemotherapy [3]. Several studies have correlated the outcome of TNBC to the tumor-immune infiltrate. These findings have led to the rationale to investigate any potential role of immunotherapies, namely immune checkpoint inhibitors (ICI), in TNBC. Recent molecular advances have identified subtypes of TNBC including basal-like, mesenchymal, luminal androgen receptor positive and immunomodulatory subtypes [4]. These subtypes are characterized by different levels of immune genes expression but all are characterized by genomic instability, high rates of genetic mutation and high rates of mutational load compared with other subtypes suggesting a potential benefit for immune-based therapies [5]. The initial trials of ICI have demonstrated potential clinical benefit and safety profile in the metastatic setting which rendered their evaluation in the earlier stage essential for the unmet need of this subgroup of patients.

\section{Immunogenic characteristics of TNBC}

Over recent years, overwhelming data have shown an important role of the immune infiltrate in the prognosis of breast cancer. While $\mathrm{CD}^{+}{ }^{+} \mathrm{T}$ cells and tumor-associated macrophages are generally associated with worse outcomes, $\mathrm{CD}^{+} \mathrm{T}$ cells have been associated with better prognosis. This has been confirmed in the clinical settings where $\mathrm{CD}^{+} \mathrm{T}$ cells seem to control human breast cancer while $\mathrm{CD} 4^{+} \mathrm{T}$ cells abrogate this action. Taken all together, this suggests that the clinical outcome of TNBC may be particularly influenced by tumor immune responses [6]. ICIs are known to enhance the antitumor function of $\mathrm{CD}^{+} \mathrm{T}$ cells, increase the ratio of $\mathrm{CD} 8^{+} \mathrm{T}$ cells to Fop3 ${ }^{+}$ $\mathrm{T}$ regulatory cells and inhibit the T regulatory cells [7]. This renders the use of ICI, particularly attractive across all the treatment settings [8].

\section{Metastatic TNBC}

The Phase I trial of pembrolizumab in TNBC, KEYNOTE-012 (NCT01848834), is a basket trial that has enrolled 111 metastatic TNBC patients that were heavily pretreated [9]. Tumor PD-L1 expression stained positive $\geq 1 \%$ in 
65 (58.6\%) patients among which 32 patients received pembrolizumab $10 \mathrm{mg} / \mathrm{kg}$ IV every 2 weeks. The objective response rate (ORR) was 18.5\% (95\% CI: 6.3-38.1\%), median progression-free survival (OS) was 1.9 months (95\% CI: 1.3-4.3 months) and median overall survival (OS) was 10.2 months (95\% CI: 5.3-17.5 months). The most common treatment-related adverse events were arthralgia (18.8\%), fatigue (18.8\%), myalgia (18.8\%), nausea (15.6\%) and diarrhea (12.5\%). Pembrolizumab was then evaluated in an open label, Phase II trial, KEYNOTE086 (NCT02447003) that included two parts. Part one enrolled TNBC patients into a cohort of treatment-naive metastatic disease or another cohort of previously treated disease with an anthracycline and a taxane in the neoadjuvant, adjuvant or metastatic setting. Part two is an expansion of the later cohort and enrolled patients with tumors strongly positive for PD-L1. All cohorts will evaluate pembrolizumab flat dose $200 \mathrm{mg}$ IV every 3 weeks until disease progression or intolerable toxicity. Pembrolizumab is also being evaluated in the Phase III trial, KEYNOTE-119 (NCT02555657), where it is compared with capecitabine, eribulin, gemcitabine and vinorelbine beyond a first line of treatment with anthracycline and/or a taxane. Another Phase III trial, KEYNOTE-355 (NCT02819518), is evaluating pembrolizumab-based combination in the first line setting of metastatic TNBC. The trial is evaluating the efficacy and safety of combining pembrolizumab with either nab-paclitaxel $(100 \mathrm{mg} / \mathrm{m} 2$ IV on days 1,8 and 15 of each 28-day cycle), paclitaxel (90 mg/m2 IV on days 1,8 and 15 of each 28-day cycle) or gemcitabine/carboplatin $(1000 \mathrm{mg} / \mathrm{m} 2$ (gemcitabine) and an area under the curve 2 (carboplatin) on days 1 and 8 of each 21-day cycle).

Atezolizumab was initially investigated in a basket trial that has enrolled 54 heavily pretreated patients with metastatic TNBC (NCT01375842) [10]. Atezolizumab was given IV at $15 \mathrm{mg} / \mathrm{kg}, 20 \mathrm{mg} / \mathrm{kg}$ or fixed dose of 1200 mg every 3 weeks. The ORR was 19\% (95\% CI: 5-42\%) and the most common adverse events were fatigue (15\%), fever (15\%) and nausea (15\%). Atezolizumab (800 mg IV on days 1 and 15) was also investigated in combination with nab-paclitaxel $\left(125 \mathrm{mg} / \mathrm{m}^{2}\right.$ IV on days 1,8 and 15$)$ on a 28 -day cycle in another Phase I trial enrolling 32 metastatic TNBC patients (NCT01633970) [11]. Interestingly, tumors lacking PD-L1 expression responded to atezolizumab albeit the responses were slightly higher in tumors defined as PD-L1-positive. Efficacy outcomes yielded an ORR of 38\% (95\% CI: 21-56\%) with a higher response rate in the first line setting. As such the Phase III trial, IMpassion130 (NCT02425891), is investigating the first line combination of atezolizumab and nab-paclitaxel in treatment-naive metastatic TNBC.

An overview of the pembrolizumab and atezolizumab data demonstrates a consistent ORR of $23-26 \%$ in the first-line treatment of metastatic TNBC but was independent of the PD-L1 expression positivity. Efficacy outcomes showed a 3-year OS reaching 100\% in patients achieving complete or partial response with either drug. However, Progression-free survival and duration of response data varied between the two drugs. Data with other ICI including nivolumab (NCT02499367, NCT02834247) and avelumab (NCT01772004, NCT02725489, NCT02484404, NCT02527434, NCT02318277) monotherapies or combinations are not yet mature for clinical use and limited to Phase I and II trials.

\section{Locally advanced TNBC}

The management of locally advanced TNBC mainly relies on neoadjuvant treatment as it allows a rapid assessment of treatment efficacy [12]. Pathologic complete response is often seen a predictor of long-term outcomes in TNBC and as such, it is the primary end point of the neoadjuvant trials [12].

Two main ICI-based combinations are undergoing prospective clinical trials. The KEYNOTE-522 trial (NCT03036488) is a randomized placebo-controlled trial enrolling patients with locally advanced TNBC that are candidates for potentially curative surgery. The patients will first be randomized to four cycles of pembrolizumab or placebo, in combination with paclitaxel and carboplatin, followed by four cycles of pembrolizumab or placebo, in combination with doxorubicin and cyclophosphamide, as neoadjuvant therapy prior to surgery. Patients will receive thereafter nine cycles of pembrolizumab or placebo adjuvant therapy postsurgery. The NeoTRIPaPDL1 trial (NCT02620280) is another Phase III trial randomizing patients with locally advanced TNBC to the neoadjuvant combination of nab-paclitaxel and carboplatin with or without atezolizumab.

Each of these Phase III trials has a different design that would clear the best position of ICI in TNBC. Other ongoing Phase II trials are ongoing with druvalumab. The first trial is testing neoadjuvant weekly nab-paclitaxel followed by dose-dense doxorubicin and cyclophosphamide with concurrent durvalumab in locally advanced TNBC (NCT02489448) and the second is evaluating the neoadjuvant durvalumab or placebo followed by nabpaclitaxel plus or minus durvalumab followed by epirubicin and cyclophosphamide plus or minus durvalumab (NCT02685059). 


\section{Early stage TNBC}

In view of the frequent relapses despite optimal local and systemic treatments, several clinical trials are ongoing to evaluate the role of ICI in the adjuvant setting. The results of these trials are eagerly awaited in view of their potential to change the current adjuvant standard of care for TNBC patients. Herein, two clinical scenarios are possible.

The first scenario is the common standard of care where TNBC patients complete upfront neoadjuvant chemotherapy followed by definitive local treatment. The patients with persistent residual disease are currently subject to two Phase III clinical trials, SWOG-21418 and A-BRAVE trials, evaluating the effect of adjuvant pembrolizumab and avelumab, respectively in TNBC patients that did not achieve pCR defined by a residual tumor $\geq 1 \mathrm{~cm}$ and/or axillary-node positive disease (NCT02954874, NCT02926196).

The second scenario is encountered less frequently as oncologists commonly opt for neoadjuvant chemotherapy in TNBC. However, there is a subset of patients that have already received definitive curative therapy with upfront surgery followed by adjuvant treatments as clinically indicated. The A-BRAVE trial included such patients to evaluate the effect of avelumab on disease-free survival (NCT02926196). Eligible patients were those who had more than four involved axillary lymph nodes and their adjuvant chemotherapy included at least three courses of an anthracycline and three courses of a taxane.

It is noteworthy that several regimens are also being evaluated in the adjuvant therapy of TNBC. This includes mainly a Phase Ib study of durvalumab and the PVX-410 vaccine as adjuvant therapy for early stage and locally advanced TNBC (NCT02826434).

\section{Conclusion \& future perspective}

In patients with TNBC, ICIs are associated with durable responses and substantial OS with a tolerable safety profile. Unfortunately, to date, none of the tested biomarkers including PD-L1 expression and tumor-infiltrating lymphocytes are suitable to reliably identify responders to ICI. The available data confirm a better patient selection for those receiving ICI in the first-line setting of metastatic TNBC.

ICI-based combinations with chemotherapy, novel targeted agents and other immunotherapies would reduce the activity of T-regulatory cells, enhance cross-penetration of tumor antigens and increase PD-L1 expression and infiltration of $\mathrm{CD}^{+} \mathrm{T}$ cells. The initial results of these trials seem to increase the response rate in metastatic TNBC and the pCR date in neoadjuvant TNBC with a tolerable safety profile. Such combinations would increase the efficacy and target population to answer the unmet needs of TNBC patients. Further trials are currently ongoing and should conclude to treatment algorithms and sequences for the management of TNBC.

\section{Financial \& competing interests disclosure}

The authors have no relevant affiliations or financial involvement with any organization or entity with a financial interest in or financial conflict with the subject matter or materials discussed in the manuscript. This includes employment, consultancies, honoraria, stock ownership or options, expert testimony, grants or patents received or pending or royalties.

No writing assistance was utilized in the production of this manuscript.

\section{References}

1 Sørlie T, Tibshirani R, Parker J et al. Repeated observation of breast tumor subtypes in independent gene expression data sets. Proc. Natl Acad. Sci. USA 100(14), 8418-8423 (2003).

2 Bauer KR, Brown M, Cress RD, Parise CA, Caggiano V. Descriptive analysis of estrogen receptor (ER)-negative, progesterone receptor (PR)-negative, and HER2-negative invasive breast cancer, the so-called triple-negative phenotype: a population-based study from the California cancer Registry. Cancer 109(9), 1721-1728 (2007).

3 Rouzier R, Perou CM, Symmans WF et al. Breast cancer molecular subtypes respond differently to preoperative chemotherapy. Clin. Cancer Res. Off. J. Am. Assoc. Cancer Res. 11(16), 5678-5685 (2005).

4 Lehmann BD, Bauer JA, Chen X et al. Identification of human triple-negative breast cancer subtypes and preclinical models for selection of targeted therapies. J. Clin. Invest. 121(7), 2750-2767 (2011).

5 Lynce F, Xiu J, Obeid E et al. Tumor mutational load in gynecological and breast cancer. Poster presented at: 2017 ASCO-SITC Clinical Immuno-Oncology Symposium. J. Clin. Oncol. 35(suppl 7S; abstr 44) (2017).

6 DeNardo DG, Brennan DJ, Rexhepaj E et al. Leukocyte complexity predicts breast cancer survival and functionally regulates response to chemotherapy. Cancer Discov. 1(1), 54-67 (2011).

7 Quezada SA, Peggs KS, Curran MA, Allison JP. CTLA4 blockade and GM-CSF combination immunotherapy alters the intratumor balance of effector and regulatory T cells. J. Clin. Invest. 116(7), 1935-1945 (2006). 
8 Stagg J, Allard B. Immunotherapeutic approaches in triple-negative breast cancer: latest research and clinical prospects. Ther. Adv. Med. Oncol. 5(3), 169-181 (2013).

9 Nanda R, Chow LQM, Dees EC et al. Pembrolizumab in patients with advanced triple-negative breast cancer: phase Ib KEYNOTE-012 Study. J. Clin. Oncol. 20; 34(21), 2460-2467 (2016).

10 Emens LA, Braiteh FS, Cassier P et al. Inhibition of PD-L1 by MPDL3280A leads to clinical activity in patients with metastatic triple-negative breast cancer. Abstract presented at: 37th Annual CTRC-AACR San Antonio Breast Cancer Symposium. Cancer Res. 75(Suppl. 15, abstr 2859) (2015).

11 Adams S, Diamond JR, Hamilton EP et al. Phase Ib trial of atezolizumab in combination with nab-paclitaxel in patients with metastatic triple-negative breast cancer (mTNBC). J. Clin. Oncol. 20; 34(15_Suppl.), 1009-1009 (2016).

12 Cleator S, Heller W, Coombes RC. Triple-negative breast cancer: therapeutic options. Lancet Oncol. 8(3), 235-244 (2007). 